\title{
A Model-Based Approach to Multi-Modal Mass Tuning of a Micro-Scale Resonator
}

\author{
David Schwartz*, Dennis Kim ${ }^{\dagger}$, and Robert M'Closkey* \\ Mechanical and Aerospace Engineering Department \\ University of California, Los Angeles
}

\begin{abstract}
The signal-to-noise ratio of axisymmetric vibratory gyroscopes is maximized when a pair of coriolis-coupled modes resonate at the same frequency. The manufacturing process of micro-scale resonators creates random minute mass and stiffness asymmetries that cause the natural frequencies of these modes to deviate from one another, thereby degrading sensor performance. One method of "tuning" these modal frequencies to equality involves using electrostatic forces to selectively soften the stiffness at points in the resonant structure. This generally requires large volume electronics that are incompatible with application requirements common to these sensors. Alternatively, modal frequency tuning by mass perturbation of the resonator is a promising approach because it is permanent and requires no ancillary electronics. In this paper, a novel micro-scale resonator is presented which lends itself to mass perturbation experiments. A resonator model, based on empirical frequency response data, is used to guide the mass perturbation process and demonstrates how multiple pairs of modes can be tuned.
\end{abstract}

\section{INTRODUCTION}

In recent years there has been a resurgence of interest in developing compact inertial sensors for use in GPS-denied environments. Before this can become a reality, though, advancements must first be made in the size and accuracy of the vibratory gyroscopes with axisymmetric resonators that are proposed for use in such systems. In theory, the symmetric nature of the resonator design enables high performance characteristics due to the natural modal degeneracy intrinsic to axisymmetric designs. Unfortunately, the manufacturing process invariably produces random, minute asymmetries in the resonant structure. These asymmetries cause the pairs of modes that are used by the gyroscope to sense angular motion to resonate at slightly different frequencies which in turn reduces the signal-to-noise ration (Fig.1) [5].

A pair of modes can be "tuned" to operate at the same frequency either by using electrostatic forces or selective mass loading. Electrostatic tuning involves applying static voltages to electrodes to change the effective modal stiffness. This process can be effective, but the electronics required to maintain the necessary voltage stability are generally larger than the overall size requirements of the sensor [6]. Thus, mass tuning, in which a pair of modes is tuned by altering the

\footnotetext{
* Email: daves@ucla.edu

† Email: dongj@seas.ucla.edu

\$ Corresponding author. Email: rtm@seas.ucla.edu. This work is supported by DARPA contract W31P4Q-10-1-0017.
}
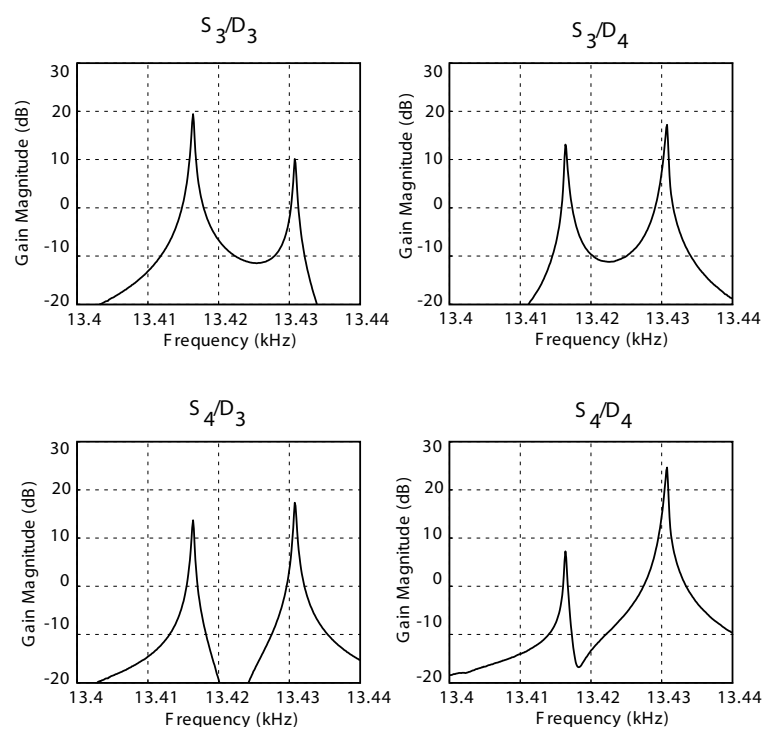

Fig. 1. The $2 \times 2$ empirical frequency response of the $n=2$ pair of modes of the UCLA Resonator (URES). If the resonator were fabricated with perfect symmetry, these modes would resonate at the same frequency and only a single peak would be observable in each channel. In the case presented here, minute asymmetries have caused the modes to exhibit a significant frequency split. A similar split occurs in the $n=3$ modal pair as presented Fig. 6.

mass distribution of the resonator, appears to be a necessary endeavor.

On its face, mass tuning can be a straightforward process. Fig. 2 displays a potential mass tuning scenario for the fundamental Corilolis-coupled $n=2$ modes of simple ring geometry. The $\mathrm{n}=2$ modes have an elliptical shape and are the ones most commonly used for rate sensing. With no asymmetry, the ring can vibrate with an $\mathrm{n}=2$ mode shape at any angular orientation with the same frequency. If a mass asymmetry exists, two modes clearly define themselves whose frequencies are no longer matched [1], [8]. The device can be tuned by placing additional mass on the anti-node of the mode with a higher frequency.

Mass tuning has been successfully implemented on larger devices. The Hemispherical Resonator Gyroscope (HRG) [7], most notably used on the Hubble Space Telescope for instrument stabilization, has been a benchmark for performance, though its $3 \mathrm{~cm}$ diameter is too large for it to be considered a micro-scale device. The HRG is tuned by perturbing its mass 

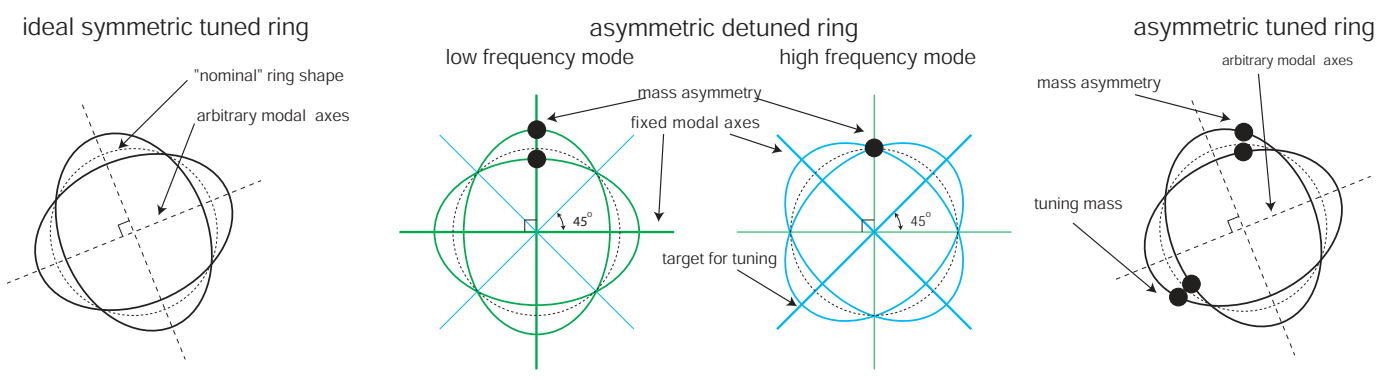

Fig. 2. A mass tuning example for a simple ring. Left:With no asymmetry, the ring can vibrate with an $\mathrm{n}=2$ mode shape at any angular orientation with the same frequency. Center: If a mass asymmetry exists, a low frequency mode defines itself with an anti-node at the location of the mass loading (green), and a low frequency mode is defined with anti-nodes 45 degrees away from that point (blue). Right: The device can be tuned by placing additional mass on the anti-node of the high frequency mode.

at points on the quartz cup rim, and although the details of the tuning process have not been publicly disclosed, they are reported to be meticulous and expensive (see [11] for analysis by another group not involved with the HRG development).

The expressions concerning the effects of mass perturbations on the modal frequencies and the positions of the modal axes on rings (which have similar modal characteristics to other axisymmetric devices) have long been derived and verified on physical systems [1], [2]. These expressions motivated the process described in Fig. 2 which was demonstrated on a MEM device when laser ablation was employed to remove mass and predictably alter the frequency split [4], [3]. Unfortunately, this process is not easily extended to a "production" environment because the determination of the location of the anti-nodes requires significant effort. We recently developed an alternative model-driven approach that appears to be quite systematic. The method was demonstrated on a planar, $11 \mathrm{~cm}$ diameter steel resonator, dubbed the Macro DRG, but is theoretically applicable to all axisymmetric resonators [10].

For this paper, the UCLA resonator (URES) was created to further demonstrate the feasibility of this concept (Fig. 3). URES employs a version of planar concentric ring design that is similar to other successful devices. In the URES design, the connections between concentric rings have been exaggerated in order to make 96 easy targets for various forms of mass perturbations. Also, the exterior electrodes, which are used for actuation, sensing, and electrostatic softening (if need be), are arranged so that they can be used to optimally measure both the $n=2$ and $n=3$ modes.

An electrode configuration which can sense both $n=2$ and $n=3$ modes is chosen for this study. The electrodes are connected to yield a four-input/four-output system. Selected channels of the empirical transfer function are fit with a 2 degree-of-freedom model and the relationship between the model and the nature of the physical asymmetry is revealed in a novel manner. The models are then validated, and finally used for modal frequency tuning with appropriate mass perturbations.

\section{EXPERIMENTAL DESIGN}

A single URES is pictured in Fig. 3, as well as an SEM image displaying some of its details. The resonator itself is $1 \mathrm{~cm}$ across and its central disk, denoted " 1 " in Fig. 3, is attached to the base wafer, while thin concentric rings " 2 " are free to resonate primarily in-plane. The exaggerated connections between pads are $150 \mu \mathrm{m}$ in diameter and are plated with gold to aid with perturbations " 4 ". The perturbations are in the form of gold balls with a mass of approximately $20 \mu \mathrm{g}$. Twenty four electrodes " 6 " surround the outermost ring and are electrically connected to the larger probe pads " 7 ".

Test signals are generated using a DSP and are provided, via the probe card, directly to the drive electrodes (Right hand side of Fig. 3). The resonator is DC biased so that, as the resonator vibrates, the gap between the resonator and the sense electrodes changes. This creates a small current that is amplified by electronics located on the probe card. The signals are filtered before being sampled by the DSP. Typically, the $\left\{D_{3}, D_{4}, S_{3}, S_{4}\right\}$ electrodes are used to perform frequency response experiments on the the $n=2$ modes because their sensors and actuators are 45 degrees apart, whereas the $\left\{D_{1}, D_{2}, S_{1}, S_{2}\right\}$ electrodes are thirty degrees apart and are used to test the $n=3$ modes. In both cases, a chirp signal that encompass the modes of interest are applied to one actuator at a time while data is recovered from the corresponding sense electrodes to create a $2 \times 2$ transfer function as displayed in Fig. 1.

\section{System Modeling}

\section{A. Frequency Domain Model}

The system identification method for guiding the mass perturbation process is based on the one developed by the authors for electrostatic tuning of the gyro dynamics [6] . For mass tuning, the linear mechanics of nearly degenerate vibratory gyros in a neighbourhood of the Coriolis-coupled modes can be modeled as

$$
Z_{\text {sens }}^{-1}(s) R H_{\text {in }}(s),
$$

where $s$ is the Laplace transform variable and where

$$
Z_{\text {sens }}(s):=M s^{2}+C s+K \text {. }
$$



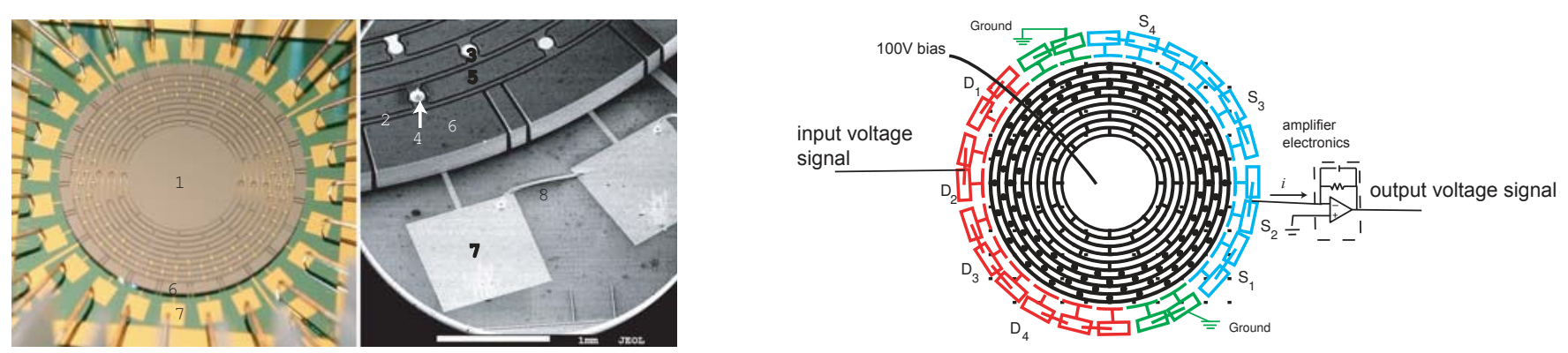

Fig. 3. Left: An example of the UCLA Resonator (URES). The central disk, 1, is attached to the base wafer, while thin concentric rings, $\mathbf{2}$, are free to resonate. The exaggerated connections between pads, $\mathbf{3}$, are plated with gold to aid with perturbations, $\mathbf{4}$. The space between rings, $\mathbf{5}$, does not resonate. The probe pads are electrically connected to the the 24 electrodes, $\mathbf{6}$, that sit around the outside of the outermost ring. The probes which connect to the signal conditioning board connect to the probe pads, 7. Right: The electrodes are paired together for sensing and actuation using wire bonds, 8. The signal flow used to generate the empirical frequency response data for the $S_{2} / D_{2}$ channel is also shown.

In this model, $M, C$ and $K$ are real $2 \times 2$ positive definite mass, damping and stiffness matrices. The angular rotation rate of the sensor is assumed to be zero in this model. The subscript on $Z_{\text {sens }}$ denotes that the system matrices are written in the generalized coordinates specified by the sensor frame. The transfer function $H_{\text {in }}$ represents any dynamics associated with the signal conditioning electronics and $R \in$ $\mathbf{R}^{2 \times 2}$ captures the effects of non-colocated pick-offs and forcers.

The model parameters $\left\{M, C, K, R, H_{\text {in }}\right\}$ are estimated by fitting frequency response data. In other words, an experiment yields two-input/two-output complex valued frequency response data $\left\{\psi_{1}, \psi_{2}, \ldots, \psi_{N_{f}}\right\}$ corresponding to the $N_{f}$ real frequencies $\left\{\omega_{1}, \omega_{2}, \ldots, \omega_{N_{f}}\right\}$.

The minimax optimization problem for estimating the sensor parameters is

$$
\min _{\substack{M>I \\ K>0, C>0 \\ R_{l} \in \mathbf{C}^{2 \times 2}, l=0,1, \ldots, n_{R}}} \max _{q=1, \ldots, N_{f}} \bar{\sigma}\left(\tilde{R}_{q}-Z_{\text {sens }}\left(j \omega_{q}\right) \psi_{q}\right),
$$

where

$$
\tilde{R}_{q}:=\sum_{l=0}^{n_{R}} R_{l} \omega_{q}^{l}
$$

and where evaluating $Z_{\text {sens }}$ at the $q$ th frequency point yields

$$
Z_{\text {sens }}\left(j \omega_{q}\right):=-M \omega_{q}^{2}+K+j C \omega_{q} .
$$

The largest singular value is denoted $\bar{\sigma}$. The constraint $M>I$ in (3) is imposed rather than the typical $M>0$ because in the latter case all of the free parameters may be scaled by a nonzero constant in order to make the cost arbitrarily small without actually changing the model frequency response. Also note that $R H_{\text {in }}$ has been replaced by $\tilde{R}$. This recognizes the fact that any additional dynamics due to, for example, signal conditioning preamplifiers, should not exhibit significant magnitude and phase changes in a neighbourhood of the resonant modes. If these dynamics can be reflected to the sensor input then they can be combined with $R$ into a low order polynomial function of frequency with coefficients in $\mathbf{C}^{2 \times 2}$, i.e. $\tilde{R}$ is degree $n_{R}$. In fact, $\tilde{R}$ can be viewed as the combination of the first few terms of the Taylor series expansion of the frequency response function of $H_{\text {in }}$ including the non-collocation effects. The details on how (3) is restated as a generalized eigenvalue problem is shown in [6], [10].

The next section shows how the generalized eigenvectors and eigenvalues of $(M, K)$ are used to estimate the location of the anti-nodes in the resonator.

\section{B. Interpretation of the Frequency Domain Model}

The unforced resonator model is $M \ddot{\mathbf{x}}+C \dot{\mathbf{x}}+K \mathbf{x}=0$, where $\mathbf{x}$ represents the vector of generalized displacements associated with the identified modes. The time response of the generalized displacements in the unforced problem can be derived assuming the initial state has zero velocity and the initial displacement is constrained to be a scalar multiple of one of the two generalized eigenvectors of $M$ and $K$. Considerable simplification is possible if damping is neglected. Under this zero damping assumption the generalized displacement vector is given by

$$
\mathbf{x}(t)=\left|\mathbf{x}_{0}\right| \mathbf{v}_{n i} \cos \left(\lambda_{n i} t\right) \quad i \in 1,2
$$

where $\mathbf{v}_{n i}$ and $\lambda_{n i}$ are the eigenvector and square root of the eigenvalue associated with the high and low frequency modes with $n$ modal diameters and $\left|\mathbf{x}_{0}\right|$ is the norm of the initial state. The index $i=1$ refers to the lower frequency mode while $i=2$ refers to the higher frequency mode. The physical measurements are proportional to radial velocity, averaged over the electrode area, so the measurement in this "experiment" is given by

$$
\mathbf{y}(t)=-\lambda_{n i}\left|\mathbf{x}_{0}\right| \mathbf{v}_{n i} \sin \left(\lambda_{n i} t\right) \quad i \in 1,2 .
$$

On the other hand, the time response $\mathbf{y}$ can also be determined from the approximate mode shape. The mode shape of the outermost ring of a URES is closely approximated by that of a simple ring, which is

$$
\begin{aligned}
& w(\theta, t)=U_{0} \alpha_{n} \cos \left(n\left(\theta-\Psi_{n i}\right)\right) \cos \left(\omega_{n i} t\right) \\
& u(\theta, t)=U_{0} \sin \left(n\left(\theta-\Psi_{n i}\right)\right) \cos \left(\omega_{n i} t\right)
\end{aligned}
$$

where $w$ and $u$ are the radial and tangential motion position with respect to nominal at a location $\theta$ on the ring [8]. As illustrated in Fig. reffig5, the angle $\Psi_{n i}$ gives the to-bedetermined position anti-nodes of a particular mode. Also, 


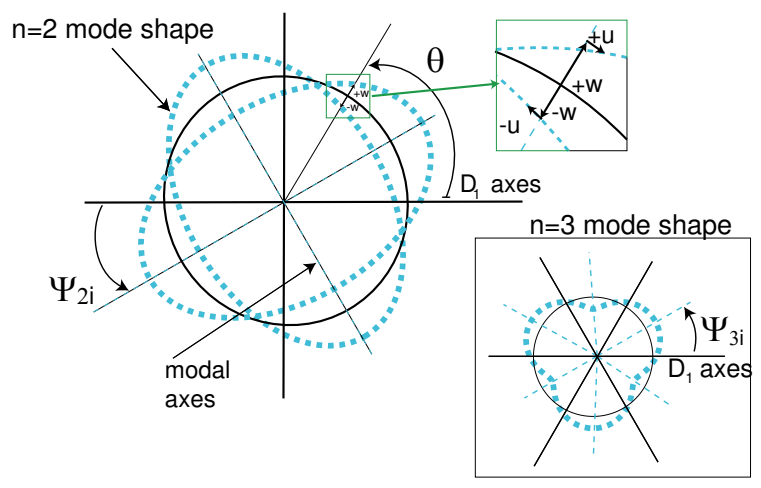

Fig. 4. Illustration of the parameters used in analysis The $D_{1}$ axes are arbitrarily chosen to initialize a coordinate system so that the $\theta$ and $\Psi$ parameters have meaning. The mode shape of the tri-lobular $n=3$ modes is also illustrated in the lower right.

$\omega_{n i}$ is the frequency of vibration, $n$ is the number of nodal diameters, $U_{0}$ is the amplitude of the tangential motion and $\alpha_{n}$ is the factor by which the radial motion is larger than the tangential motion. Thus, the expression for $\mathbf{y}$ can also be expressed as

$$
\begin{aligned}
\mathbf{y}(t) & =\left[\begin{array}{l}
\frac{d w\left(\theta_{1}, t\right)}{d t} \\
\frac{d w\left(\theta_{2}, t\right)}{d t}
\end{array}\right] \\
& =-\omega_{n i} \alpha_{n} U_{0}\left[\begin{array}{ll}
\cos \left(n\left(\theta_{1}-\Psi_{n i}\right)\right) \\
\cos \left(n\left(\theta_{2}-\Psi_{n i}\right)\right)
\end{array}\right] \sin \left(\omega_{n i} t\right) \\
& =-\omega_{n i} \alpha_{n} U_{0}\left[\begin{array}{ll}
\cos \left(n \theta_{1}\right) & \sin \left(n \theta_{1}\right) \\
\cos \left(n \theta_{2}\right) & \sin \left(n \theta_{2}\right)
\end{array}\right]\left[\begin{array}{l}
\cos \left(n \Psi_{n i}\right) \\
\sin \left(n \Psi_{n i}\right)
\end{array}\right] \sin \left(\omega_{n i} t\right)
\end{aligned}
$$

where $\theta_{1}$ and $\theta_{2}$ are the angular locations of the two sense electrodes. Equating (8) and (6), and setting $\lambda_{n i}=\omega_{n i}$, yields an expression from which $\Psi_{n i}$ is found:

$$
\begin{aligned}
{\left[\begin{array}{c}
\cos \left(n \Psi_{n i}\right) \\
\sin \left(n \Psi_{n i}\right)
\end{array}\right] } & =-\frac{\left|\mathbf{x}_{0}\right|}{\alpha_{n} U_{0}}\left[\begin{array}{lr}
\cos \left(n \theta_{1}\right) & \sin \left(n \theta_{1}\right) \\
\cos \left(n \theta_{2}\right) & \sin \left(n \theta_{2}\right)
\end{array}\right]^{-1} v_{n i} \\
\Longrightarrow \Psi_{n i} & =\frac{1}{n} \tan ^{-1}\left(\frac{\left[\begin{array}{ll}
-\cos \left(n \theta_{2}\right) & \cos \left(n \theta_{1}\right)
\end{array}\right] v_{n i}}{\left[\begin{array}{ll}
\sin \left(n \theta_{2}\right) & -\sin \left(n \theta_{1}\right)
\end{array}\right] v_{n i}}\right)
\end{aligned}
$$

Thus, one can find an estimate for the angular orientation of both the low and high frequency anti-nodes from the identified mass and stiffness matrices.

\section{Mass Perturbation Model}

Once the orientation of the anti-nodes and the modal frequency split have been identified, it is possible to predict the change in orientation and split after a point mass perturbation is performed using the results of [8]. The perturbed frequency $\omega_{n i}$ of a mode with a particular orientation defined by $\Psi_{n i}$ is defined by the ratio

$$
\begin{aligned}
\frac{\omega_{n 0}^{2}}{\omega_{n i}^{2}}= & 1+\gamma_{n 1} \Sigma_{i=1}^{N_{m}} m_{n i}+ \\
& \frac{\gamma_{n 2}}{\omega_{n 0}}\left(\cos \left(2 n \Psi_{n i}\right) \sigma_{\cos }+\sin \left(2 n \Psi_{n i}\right) \sigma_{\sin }\right)
\end{aligned}
$$

where

$$
\begin{aligned}
& \sigma_{\mathrm{cos}}=\Sigma_{a=1}^{N_{a}} m_{a} \cos \left(2 n \phi_{a}\right) \\
& \sigma_{\sin }=\Sigma_{a=1}^{N_{a}} m_{a} \sin \left(2 n \phi_{a}\right)
\end{aligned}
$$

and the asymmetry is defined by $N_{a}$ point masses $m_{a}$ occurring at locations $\phi_{a}$. The variables $\gamma_{n 1}$ and $\gamma_{n 2}$ are gains that change depending on the geometry and may be determined experimentally, and $\omega_{n 0}$ is the unperturbed resonant frequency. Thus, the frequency of vibration for a particular perturbation scenario are only a function of the anti-node orientation. The maximum and minimum frequencies of this function are the modal frequencies of the resonator, while the orientations at which they occur define the corresponding mode shapes. These extremes occur when

$$
\begin{gathered}
\sin \left(2 n \Psi_{n i}\right) \sigma_{\cos }=\cos \left(2 n \Psi_{n i}\right) \sigma_{\sin } \\
\Longrightarrow \Psi_{n i}=\frac{1}{2 n} \tan ^{-} 1\left(\frac{\sigma_{\sin }}{\sigma_{\cos }}\right)+(i-1) \frac{\pi}{2 n} \quad i \in 1,2 .
\end{gathered}
$$

The ratio (10) is minimized (and the resonant frequency maximized) when $i=1$, whereas the resonant frequency reaches its maximum when $i=2$.

Next, the frequency split can be found in terms of the asymmetry:

$$
\begin{aligned}
\omega_{n 2}^{2}-\omega_{n 1}^{2}= & \frac{-2 \gamma_{n 2} \omega_{n 1}^{2} \omega_{n 2}^{2}}{\omega_{n 0}^{3}}\left(\cos \left(2 n \Psi_{n 2}\right) \sigma_{\cos }+\sin \left(2 n \Psi_{n 2}\right) \sigma_{s i n}\right) \\
& \Longrightarrow \omega_{n 2}-\omega_{n 1} \approx \gamma_{n 2} \sqrt{\sigma_{\cos }^{2}+\sigma_{\sin }^{2}}
\end{aligned}
$$

Lastly, one can calculate $\sigma_{\cos }$ and $\sigma_{\text {sin }}$ using the frequency split and the location of the high frequency anti-nodes:

$$
\begin{aligned}
& \sigma_{\cos }=\frac{\left(\omega_{n 2}-\omega_{n 1}\right) \cos \left(2 n \Psi_{n 2}\right)}{\gamma_{n 2}} \\
& \sigma_{\sin }=\frac{\left(\omega_{n 2}-\omega_{n 1} \sin \left(2 n \Psi_{n 2}\right)\right.}{\gamma_{n 2}} .
\end{aligned}
$$

\section{Tuning Methods}

A single modal frequency can be modified by finding a combination of perturbations of mass $m_{p}$ placed at locations $\phi_{p}$ that solve

$$
\begin{aligned}
& \sigma_{\cos n(0)}+\sum_{p=1}^{N m} m_{p} \cos \left(2 n \phi_{p}\right)=0 \\
& \sigma_{\sin n(0)}+\sum_{p=1}^{N m} m_{p} \sin \left(2 n \phi_{p}\right)=0
\end{aligned}
$$

where $\sigma_{\cos n(0)}$ and $\sigma_{\sin n(0)}$ are defined by the mass asymmetry state before any perturbations are made. This set of equations can be solved with any number of perturbations. A single mass solution is attained with $m_{1}=\frac{1}{\gamma_{n 2}} \sqrt{\sigma_{\text {cos }}^{2}+\sigma_{\text {sin }}^{2}}$ and $\phi_{1}=\Psi_{n 2}$. In other words, a specific quantity of mass is placed precisely at the location of the anti-node of the high frequency mode.

If there are constraints on where the mass perturbations can physically take place on the resonator, as is the case with the URES device, then two masses are required for tuning. There are multiple two mass solutions, and one simple method for finding $a$ solution is to pick two locations, denoted $\phi_{1}$ and $\phi_{2}$, where the perturbations are to occur and use

$$
\left[\begin{array}{l}
m_{1} \\
m_{2}
\end{array}\right]=\left[\begin{array}{ll}
\cos \left(2 n \phi_{1}\right) & \cos \left(2 n \phi_{2}\right) \\
\sin \left(2 n \phi_{1}\right) & \sin \left(2 n \phi_{2}\right)
\end{array}\right]^{-1}\left[\begin{array}{c}
\sigma_{\cos n(0)} \\
\sigma_{\sin n(0)}
\end{array}\right]
$$

to compute $m_{1}$ and $m_{2}$. Generally, there are two constraints on the choices of $\phi_{1}$ and $\phi_{2}$. First, they must be chosen so that the inverted matrix is nonsingular, or, equivalently

$$
\bmod \left(\phi_{1}-\phi_{2}, \frac{\pi}{2 n}\right) \neq 0 \text {. }
$$




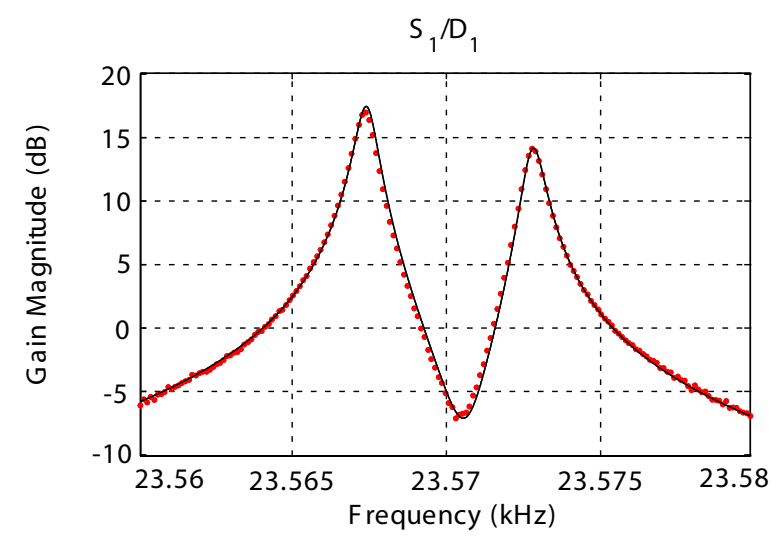

Fig. 5. Example of the model fitting algorithm. The red points display the measured frequency response magnitude, while the trace is generated using the frequency domain model using $2 \times 2$ positive definite mass, stiffness and damping matrices.

Also, the solutions $m_{1}$ and $m_{2}$ must be positive (or negative in the case mass reduction). This is guaranteed if

$$
\phi_{1} \leq \Psi_{n 2} \leq \phi_{2}, \quad 2 n \phi_{2}-2 n \phi_{1}<\pi,
$$

which is interpreted as choosing $\phi_{1}$ and $\phi_{2}$ to be on either side of the high frequency anti-node. The closer the two perturbation locations actually are to the higher frequency anti-node, the smaller the amount of mass will be required for tuning.

It is also possible to extend this method to manipulating the frequencies of multiple modal pairs. In our case, we are interested in tuning the $n=2$ and $n=3$ modes:

$$
\begin{aligned}
& \sigma_{\cos 2(0)}+\sum_{p==1}^{N m} m_{p} \cos \left(4 \phi_{p}\right)=0 \\
& \sigma_{\cos 2(0)}+\sum_{p=1}^{N m} m_{p} \sin \left(4 \phi_{p}\right)=0 \\
& \sigma_{\cos 3(0)}+\sum_{p==1}^{N m} m_{p} \cos \left(6 \phi_{p}\right)=0 \\
& \sigma_{\cos 3(0)}+\sum_{p=1}^{N m} m_{p} \cos \left(6 \phi_{p}\right)=0
\end{aligned}
$$

In general, this system of equations can be solved with two or more masses [9].

\section{EXPERIMENTAL RESUlts}

\section{A. Validation of Models}

An example of the model fit generated by using the method discussed in Section III.A is shown in Fig. 5. Only the magnitude of the transfer function and its corresponding fit are shown. Still, the model for $M, C, K$, and $R$ is a close fit for both magnitude and phase in all four channels and is more than $95 \%$ accurate as quantified by the $H_{2}$ norm of the residual.

The validation of the modelling process as well as estimates of $\gamma_{22}$ and $\gamma_{32}$ are done experimentally on a device with results shown in Table I. If a single mass perturbation is made to a measured system, the imbalance parameters $\sigma_{\cos (p)}$ and $\sigma_{\sin (p)}$ can be calculated using

$$
\begin{aligned}
& \sigma_{\cos n(p)}=\sigma_{\cos n(p-1)}+\gamma_{n 2} m_{p} \cos \left(2 n \phi_{p}\right) \\
& \sigma_{\sin n(p)}=\sigma_{\sin n(p-1)}+\gamma_{n 2} m_{p} \sin \left(2 n \phi_{p}\right) .
\end{aligned}
$$

TABLE I

PERTURBation Model VAlidation

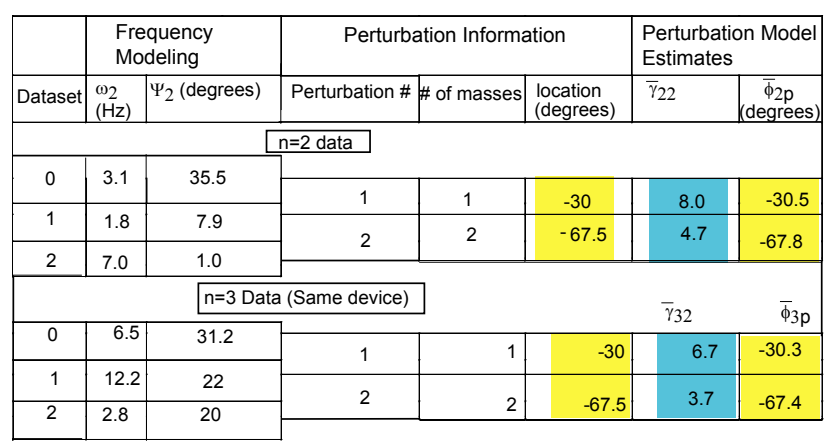

In order to validate the model, data is taken before and after a mass perturbation of known mass is made. The sensitivity $\gamma_{n 2}$ and an estimate of the location of the mass perturbation are then calculated using

$$
\begin{aligned}
& \bar{\gamma}_{n 2}=\frac{1}{m_{p}} \sqrt{\left(\sigma_{\cos n(p)}-\sigma_{\cos n(p-1)}\right)^{2}+\left(\sigma_{\sin n(p)}-\sigma_{\sin n(p-1)}\right)^{2}} \\
& \bar{\phi}_{n p}=\frac{1}{2 n} \tan ^{-1}\left(\frac{\sigma_{\sin n(p)}-\sigma_{\sin n(p-1)}}{\sigma_{\cos n(p)}-\sigma_{\cos n(p-1)}}\right)
\end{aligned}
$$

where the bar represents an estimate being made. Table I shows that the sensitivity of the mass perturbation is actually extremely variable from test to test. This can be explained by the large variability in the amount of mass being added as a consequence of the physical process that is employed to deposit the mass. The perturbation model does accurately estimate the angle at which the mass was placed. This means that it also accurately estimates the location of the high frequency mode and will be a useful aid in tuning.

\section{B. Tuning Example}

Due to the lack of a consistent mass quantum that can be placed on the resonator, tuning a modal pair to degeneracy is difficult. Despite this challenge, in this section we successfully demonstrate the model driven approach to tuning, starting with an unperturbed URES resonator. The following example uses several different tuning strategies at different stages to reach a final, modally tuned, state. At first, masses are placed with the goal of tuning both modes. As this becomes more difficult the strategy is shifted toward achieving a tuned state for one mode.

The experimental results are displayed in Table II. Each row of table gives the modeled parameters of the $n=2$ and $n=3$ models, and the location(s) of the perturbation(s) made before the data was taken. In the first stage, masses are placed in order to reduce the split in the $n=3$ mode as much as possible without reducing the split of the $n=2$ modes. Since the mass of each perturbation is so variable, the perturbations are made one at a time for the first 3 perturbations. The splits of both modes reduce each time, again confirming that the model based predictions are useful. After the fourth perturbation, the split is $1.2 \mathrm{~Hz}$ for the $n=3$ modes and 1.7 $\mathrm{Hz}$ for the $n=2$ modes. At this point (Dataset 5), the gold ball mass perturbation is too large to reduce either split with 
TABLE II

MASS TUNing EXAMPLE

\begin{tabular}{|c|c|c|c|c|c|}
\hline $\begin{array}{l}\text { Data } \\
\text { set }\end{array}$ & $\begin{array}{c}\text { Frequency Split } \\
(\mathrm{Hz})\end{array}$ & $\begin{array}{c}\text { Angle Estimate } \\
\text { (degrees) }\end{array}$ & $\begin{array}{c}\text { Frequency Split } \\
(\mathrm{Hz})\end{array}$ & $\begin{array}{c}\text { Angle Estimate } \\
\text { (Degrees) }\end{array}$ & $\begin{array}{c}\text { Deposition } \\
\text { Location } \\
\text { (Degrees) }\end{array}$ \\
\hline 1 & 14.1 & 36.2 & 8.2 & 23.1 & \\
\hline 2 & 12.3 & 39.6 & 7.0 & 34.2 & 22.5 \\
\hline 3 & 8.5 & 48.1 & 4.6 & 39.6 & 37.5 \\
\hline 4 & 5.1 & 39.5 & 2.9 & 28.5 & 37.5 \\
\hline 5 & 1.7 & 39.2 & 1.2 & 47.5 & $-37.5,30$ \\
\hline 6 & 0.46 & 34.0 & 5.7 & 38.0 & $-30,22.5$ \\
\hline 7 & 0.07 & 84.7 & 6.1 & 53.7 & fine tuning w/ink \\
\hline
\end{tabular}

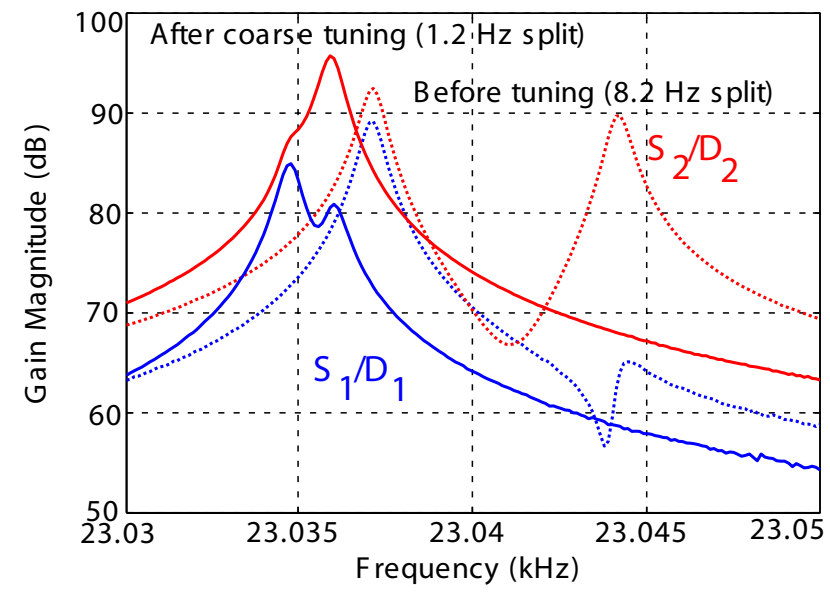

Fig. 6. Before and after tuning of the $n=3$ modes

only one mass, i.e. a single gold ball will cause "overshoot" of the desired frequencies, so any subsequent perturbations will only address reducing the $n=2$ modal split and for this case, a two-location solution is found that reduces the split of the $n=2$ modes to $0.46 \mathrm{~Hz}$ (Dataset 6). After Dataset 6 , further reduction in the $n=2$ modal frequency split is not possible with gold ball mass perturbations so a different mechanism employing silver ink deposition is used to accomplish fine tuning. The mass quantum associated with the ink is several orders of magnitude smaller than the gold balls. This process took several iterations which have been removed for brevity but the resonator's $n=2$ modes were tuned to less than a $0.1 \mathrm{~Hz}$ split (see Fig. 7) after the conclusion of the ink deposition (Dataset 7).

\section{CONCLUSION}

A model based approach has been developed and used to guide the mass loading of a micro-resonator with the objective of reducing the modal frequency split between two nominally degenerate modes. Of particular interest are the $n=2$ and $n=3$ modal pairs since these are the modes that can be exploited for angular rotation rate sensing in axisymmetric vibratory gyros. The algorithm only requires knowledge of the mode shapes in order to estimate the location of the anti-nodes from models fit to empirical frequency response data. The biggest challenge in this study was not performing the measurements or implementing the algorithm, but rather the process for effecting the mass perturbation is not sufficiently developed to produce a consistent

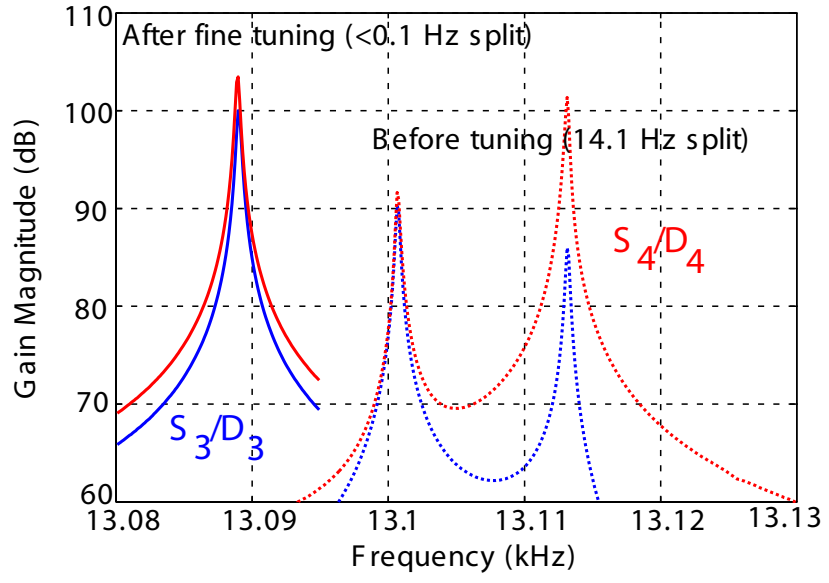

Fig. 7. Before and after tuning of the $n=2$ modes. The final modal split is less than $0.1 \mathrm{~Hz}$.

mass "quanta" across all experiments. This is especially true of the gold ball deposition which had the advantage of creating a large change in the modal properties of the resonator. Ultimately, a hybrid approach was used in which silver ink deposition was employed to further reduce the modal frequency split. The impact of mass perturbation on the modal quality factors is also of interest since higher quality factors produce higher signal-to-noise ratios in tuned vibratory gyros. Fortunately, the current data suggest that the quality factors are not changed by these mass perturbations.

\section{REFERENCES}

[1] Allaei, D., Soedel, W., and Yang, T.Y., "Natural frequencies of rings that depart from perfect axial symmetry." Journal of Sound and Vibration, Vol. 111, pp. 9-27, 1986.

[2] Charnley, T., and Perrin, R., "Perturbation studies with a thin circular ring." Acustica, Vol. 28, pp. 140-146, 1973.

[3] Fell, C.P., "Method for matching vibration mode frequencies on a vibrating structure" US Pat. 5739410, 1996.

[4] Gallacher, B. J., "Multi-modal tuning of a ring gyroscope using laser ablation" Proc. Inst. Mech. Eng. C.,Vol. 217, pp. 557-76, 2000.

[5] Kim, D., and M'Closkey, R. T., "Dissecting tuned MEMS vibratory gyros," book chapter in Feedback Control of MEMS to Atoms. Gorman, Jason J.; Shapiro, Benjamin (ed.), October 2011, Springer.

[6] Kim, D-J., and M'Closkey, R.T., "A systematic method for tuning the dynamics of electrostatically actuated vibratory gyros," IEEE Trans. Control System Technology, Vol. 14, No. 1, pp. 69-81, 2006.

[7] Lynch, D.D. "Coriolis Vibratory Gyros," Symposium Gyro Technology, Stuttgart Germany, September 1998.

[8] Rourke, A.K., McWilliam S., and Fox, C.H.J., "Multi-mode trimming of imperfect rings," Journal of Sound and Vibration, Vol. 248, No. 4, pp. 695724, 2001

[9] Schwartz, D., "Mass Perturbation Techniques for Tuning and Decoupling of a Disk Resonator Gyroscope." PhD thesis. University of California, Los Angeles, 2010.

[10] Schwartz, D., Kim, D.J., and M'Closkey, R.T., "Frequency Tuning of a Disk Resonator Gyro Via Mass Matrix Perturbation", ASME Journal of Dynamic Systems, Measurement, and Controls, Vol. 131, No. 6, p. 061004,2009 .

[11] Zhbanov, Yu. K., and Zhuravlev, V. F., "On the Balancing of a Hemispherical Resonator Gyro," Mech. Solids, Vol. 33, No. 4, pp. 213, 1998. 